\title{
Effect of Alkyl Chain Length on Adsorption and Release of Hydrophobic Drug to/from Hydrophobically-modified Gelatin Hydrogel
}

\author{
Yoshiki FuKuHARA, Yshihiro OHZUnO, Takayuki TAKEI*, and Masahiro YoshIDA \\ Department of Chemical Engineering, Graduate School of Science and Engineering, Kagoshima University, 1-21-40 Korimoto, \\ Kagoshima 890-0065, Japan
}

\begin{abstract}
Hydrogels have become popular as drug carriers. Controlled release of the drugs from hydrogels can reduce dosage, inducing prevention of side effects. However, the hydrophilicity of hydrogels interferes with controlled release of hydrophobic drugs such as anticancer agents or antibiotics. In this study, we developed hydrophobically-modified gelatin (HMG) hydrogel, which was cross-linked only by hydrophobic interaction. HMG does not require toxic chemical cross-linkers to form hydrogel. In addition, the HMG hydrogel has hydrophobic chambers in its structure which hydrophobic drugs can adsorb to and desorb from. In order to control the amount of hydrophobic drugs adsorbed into the hydrogel, hydrophobic alkyl chains with different lengths (C4-C12) were incorporated into gelatin molecules. Uranine was used as a model for hydrophobic drugs. The adsorption test exhibited that the amount of uranine adsorbed in HMG hydrogels could be controlled by varying hydrophobic alkyl chain length and that the drug could be released in a controlled manner. These results show that HMG hydrogels are promising carriers of hydrophobic drugs.
\end{abstract}

\section{Introduction}

Many compounds have been developed as candidates of practical drugs, but they are rarely used in medical practice. It is because the concentration of the drugs at the target sites is generally low owing to rapid diffusion by vasculature after administration (Kidane and Bhatt, 2005). In order to obtain an effective concentration using poorly bioavailable drugs, large doses of the drug are required. With a large dose, some therapeutic effects can be expected, but it causes serious side effects and drug retention in the body (Senapati et al., 2018).

In recent years, drug delivery systems (DDS) have attracted much attention. DDS refers to delivering the drug in the required amount, for the required time, and at the required place. The advantages of DDS induce reduction of the dose and frequency of administration of the drug, resulting in decrease in side effects at non-target sites. Furthermore, this helps to reduce medical expenses. In DDS, the drugs are incorporated into polymer network to be released from the material in a predetermined manner. So far, various synthetic polymers and natural polymers have been utilized as drug carriers (Nosrati et al., 2019; Yahia et al., 2019; Mauri et al., 2019).

Hydrogels are a three-dimensional network composed of cross-linked hydrophilic polymers that can absorb a high percentage of water (Zhou et al., 2011). The elasticity of the hydrogel minimizes irritation to surrounding tissue after implantation. In addition, low interfacial tension between the hydrogel surface and the body fluid minimizes protein adsorption, leading to low cell adhesion and reduced frequency of negative immune responses to the hydrogels (Bhattarai et al., 2010). Because of these biological advantages, hydrogels have been used as biomaterials for a wide range of applications such as tissue engineering and regenerative medicine (Saghazadeh et al., 2018). Furthermore, the unique physical properties of hydrogels have attracted interest for applications as a drug carrier. By controlling the density of cross-linking sites in the gel matrix and hydrophilicity of polymers, highly porous structures can be easily prepared (Hoare et al., 2008). Their porosity allows for loading of the drug into the gel matrix and controlling its release rate. However, it is difficult to achieve controlled delivery of hydrophobic drugs by hydrogel. The reason is that it is generally difficult to incorporate the hydrophobic drugs into hydrophilic hydrogels (Larrañeta et al., 2018). Hydrophobic drugs include many important drugs such as anti-cancer drugs and antibiotics ( $\mathrm{Li}$ et al., 2014). Therefore, many hydrogels have been developed as carriers of hydrophobic drugs (Nitta et al., 2016; Yan et al., 2019). However, most of them require chemical crosslinkers or polymerization initiators, which may be harmful to the living body.

Our purpose is to develop a toxic chemical crosslinker-free gelatin hydrogel that can be delivered in the bodies by surgical implantation and have an ability to control the release of hydrophobic drug. Gelatin is a natural polymer derived from collagen and has been widely used in medical materials for its biodegradability

* Corresponding author: takei@cen.kagoshima-u.ac.jp 
and biocompatibility (Young et al., 2005). In addition, gelatin has many reactive functional groups and is easy to modify. In this study, we developed a stable hydrogel composed of hydrophobically-modified gelatin (HMG). The hydrogel can be prepared without using toxic chemical cross-linkers because hydrophobic interaction exerted between hydrophobic segment of each molecules acts as cross-linking sites. Furthermore, this hydrogel has hydrophobic chambers in its structure which hydrophobic drugs can adsorb to and desorb from.

In this study, alkyl groups of various lengths were introduced into gelatin by the nucleophilic reaction of amines. We investigated the effect of alkyl chain length on the swelling ratio of hydrogels, the amount of hydrophobic drugs adsorbed and their release behaviour.

\section{Experimental}

\subsection{Materials}

Gelatin (type B) was purchased from Sigma-Aldrich (Saint Louis, USA). Butanal, hexanal, octanal, dodecanal and uranine were purchased from Wako Pure Chemical Industries (Osaka, Japan). 2-Picoline borane was purchased from Junsei Chemical (Tokyo, Japan).

\subsection{Synthesis of HMGs}

Four types of HMG with alkyl groups of different lengths were prepared by the reaction between fatty aldehydes and primary amino groups of gelatin. The fatty aldehydes used were butyraldehyde (C4), hexanal (C6), octanal (C8) and dodecanal (C12). First, gelatin (7 g) was added to 233 $\mathrm{ml}$ of $\mathrm{Ca}^{+}$and $\mathrm{Mg}^{2+}$ free phosphate buffered saline (CMFPBS, pH 7) and heated to dissolve. Then, $167 \mathrm{ml}$ of ethanol was added to this solution. Fatty aldehydes were dissolved in a small amount of ethanol that was then added to the gelatin solution. In order to achieve complete substitution, the added molar amount of fatty aldehyde was 5 times the amino groups of gelatin. This solution was stirred at room temperature for 6 hours to form a Schiff base between the fatty aldehyde and the primary amine. Then, 2-picoline borane was added to this solution and reacted with stirring (24 hours) to reduce an unstable Schiff base double bond. The HMG solution was poured into $1.2 \mathrm{~L}$ of ethanol and precipitated. The precipitate of HMG was washed three times with $400 \mathrm{ml}$ of ethanol to remove unreacted fatty aldehyde and 2-picoline borane. HMG particles were obtained by centrifugation and vacuum drying. The hydrophobic group modification rate of HMG was measured by the TNBS method (Morcol et al., 1997; Yoshizawa and Taguchi, 2014).

\subsection{Preparation of HMG hydrogels}

HMG was dissolved to dimethyl sulfoxide (DMSO) at $10 \%(\mathrm{w} / \mathrm{v})$ by heating and stirring. The HMG solution was poured into a 12 well plate and frozen at $-20^{\circ} \mathrm{C}$ for 6 hours.
Subsequently, DMSO was removed using a lyophilizer to obtain a porous dry gel. The dry gel was immersed in ethanol and degassed. Then, it was added to CMF-PBS to displace the solvent in the gel.

\subsection{Swelling ratio}

HMG hydrogels with different alkyl chain lengths were tested. The dry gels were weighed and the hydrogels were prepared as described above. The HMG hydrogels were immersed in CMF-PBS and removed after 2 hours. Excess CMF-PBS on surface of the hydrogels were removed using filter paper. The samples were weighed after equilibrium swelling. The swelling ratio was calculated by Eq. (1).

$$
\text { Swelling ratio }=\left(\frac{W_{s}-W_{d}}{W_{d}}\right) \times 100
$$

where $W_{d}(\mathrm{~g})$ and $W_{s}(\mathrm{~g})$ represent the dry and wet weight of the HMG hydrogel, respectively.

\subsection{Adsorption test}

The hydrophobic drug adsorption performance of the HMG hydrogel was studied by batch testing. Uranine was used as a model for hydrophobic drugs. The HMG hydrogels swollen with CMF-PBS were immersed in 2.5 $\times 10^{-5} \mathrm{~mol} / \mathrm{L}$ uranine solution $(4 \mathrm{~mL})$ and shaken $(130$ $\mathrm{rpm})$ at $4{ }^{\circ} \mathrm{C}$ for 24 hours to achieve adsorption equilibrium. The dry weight of the hydrogel was $0.03 \mathrm{~g}$. The uranine concentration was determined by measuring the fluorescence intensity using a flexible microplate reader (Infinite M200 FA, Wako Pure Chemical Industries, Osaka). The excitation wavelength of uranine was $485 \mathrm{~nm}$ and the fluorescence wavelength was $535 \mathrm{~nm}$. The adsorption capacity $\boldsymbol{q}$ (mol-uranine/g-gelatin) of uranine to the HMG hydrogel was calculated based on the difference in concentrations between the initial and final solution. $\boldsymbol{q}$ was expressed by Eq. (2).

$$
q=\frac{\left(C_{0}-C_{e}\right) V}{m}
$$

where $C_{0}(\mathrm{~mol} / \mathrm{L})$ and $C_{e}(\mathrm{~mol} / \mathrm{L})$ indicate the initial and equilibrium uranine concentrations, respectively. $V(\mathrm{~L})$ is the volume of the uranine solution and $m(\mathrm{~g})$ is the dry weight of the HMG hydrogel.

\subsection{Desorption test}

The desorption of uranine from HMG hydrogel (C12) was investigated by immersing loaded hydrogel in fresh $0.6 \mathrm{ml}$ of CMF-PBS. The solution was withdrawn over time and the amount of uranine desorbed from the hydrogel was determined by a microplate reader. In order to reproduce the body fluid clearance by organs in the bodies, the solution was replaced every 6 hours with fresh CMF-PBS. 
(A)
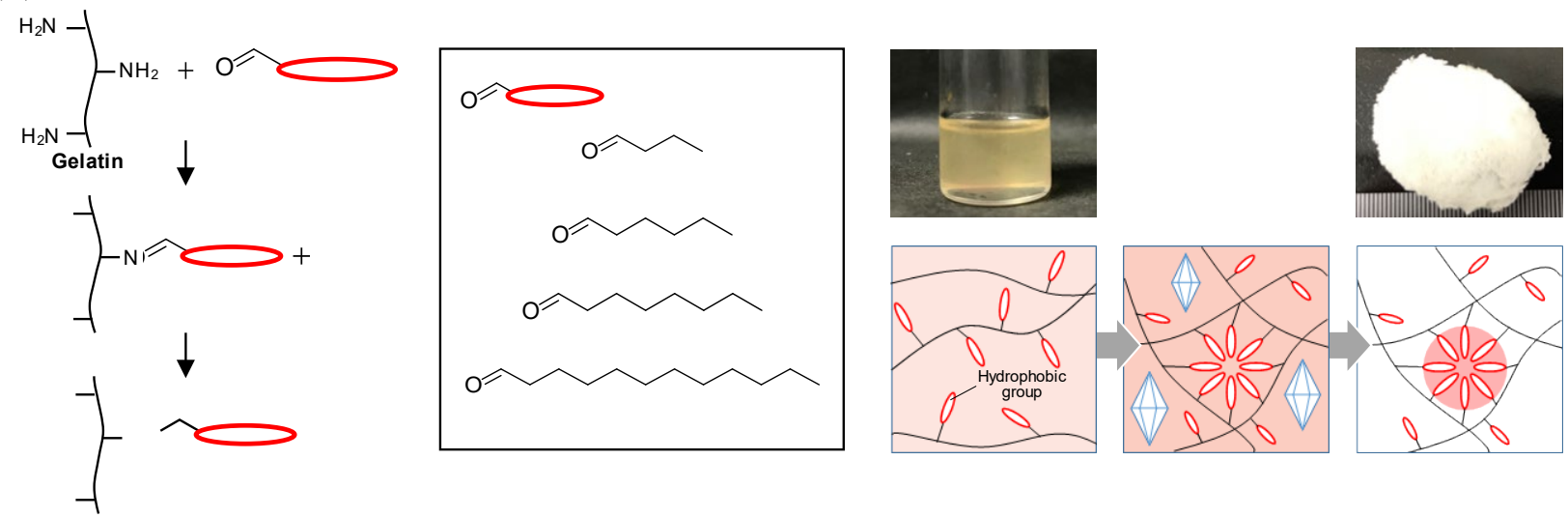

Figure 1. (A) Nucleophilic substitution reaction between amino groups of gelatin molecules and fatty aldehyde. (B) Possible crosslinking sites of HMG hydrogel.

Cumulative uranine release $C R(\%)$ was calculated as the ratio of the desorption amount at the time $t$ to the adsorption amount before release. $C R$ was given by Eq. (3).

$$
C R=\frac{C_{t} V_{t}+\sum C_{n} V_{n}-C_{e} V_{e}}{q_{e}} \times 100
$$

where $C_{t}(\mathrm{~mol} / \mathrm{L})$ and $V_{t}(\mathrm{~L})$ respectively represent the concentration of uranine at the time of sampling and the solution volume. Further, $C_{e}(\mathrm{~mol} / \mathrm{L})$ and $V_{e}(\mathrm{~L})$ represent the concentration and volume of the uranine solution held by the HMG hydrogel before release. $q_{e}$ (mol) is adsorption amount of uranine before release. $\sum C_{n} V_{n}(\mathrm{~mol})$ is the amount of uranine extracted by sampling.

\section{Results and Discussion}

\subsection{Synthesis of HMGs and preparation of HMG hydrogels}

In this study, HMG with different alkyl chain lengths were synthesized (Figure 1A). The modification rate of the hydrophobic group was measured by quantifying amino groups of gelatin by the TNBS method (Table 1). All obtained HMG had a high modification rate of more than $90 \%$. The modification rate slightly decreased as the alkyl chain length increased. This is because a fatty aldehyde with longer alkyl chain behaves as a steric hindrance by itself, resulting in decrease in contact frequency with the amino groups. Figure 1B shows possible physical crosslinking sites of HMG hydrogel. By freezing the $\mathrm{HMG}$ solution, solid crystals of DMSO would form and grow,

Table 1. Conditions for synthesizing HMG.

\begin{tabular}{ccc}
\hline $\begin{array}{c}\text { Name } \\
\text { (alkyl chain length) }\end{array}$ & $\begin{array}{c}\text { Feeding ratios of fatty aldehyde } \\
\text { to amino groups of gelatin }\end{array}$ & Modification (\%) \\
\hline C4 & 5 & 99 \\
C6 & 5 & 99 \\
C8 & 5 & 98 \\
C12 & 5 & 90 \\
\hline
\end{tabular}

while excluding the HMG molecules as an impurity. The phase separation results in the generation of HMG-rich solution phases in the solution. In the HMG-rich solution phase, hydrophobic groups of HMG assemble with each other by hydrophobic interaction to form physical crosslinking sites. Porous dry gels are obtained by removing DMSO from the constructs by drying.

\subsection{Swelling ratio}

The swelling ratio of hydrogels is an important factor for drug delivery applications because it has a large effect on diffusion rate of drugs and their flexibility. The difference in the appearance of the gels and the swelling ratio are shown in Figure 2. All samples showed good swelling properties of $600-1200 \%$. The swelling ratio decreased as alkyl chain length increased. The swelling ratio was well correlated with alkyl chain length of HMG. This is because as alkyl chain length increases, hydrophobic interactions acting between the molecules become

(A)

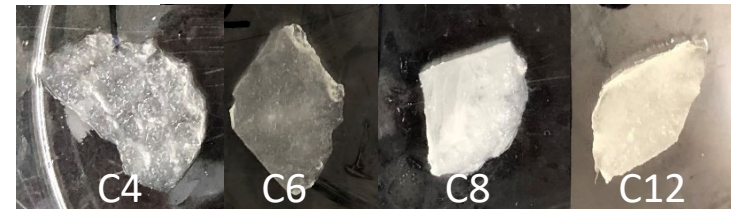

(B)

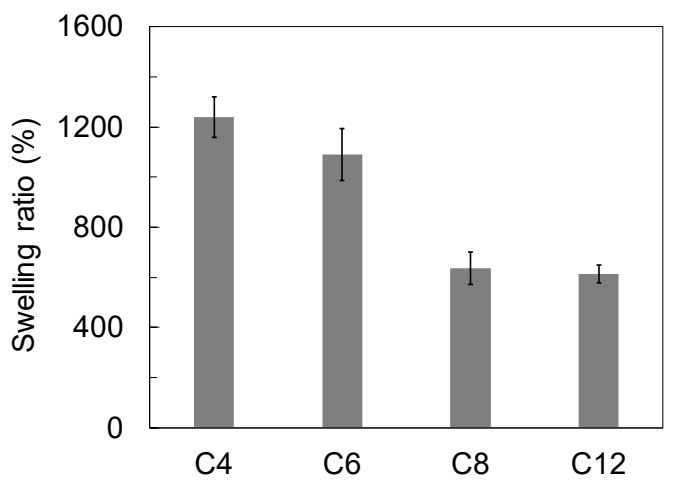

Figure 2. Images (A) and swelling ratio (B) of $\mathrm{HMG}$ hydrogels with different alkyl groups $(n=3)$. 
stronger. As a result, the HMG molecules come close to each other, and form denser polymer network. Another factor affecting the swelling ratio may include hydrophilicity of polymer. As the alkyl chain length increased, the affinity of the polymer to water decreases, and amount of water molecules binding to HMG molecules decreases. The swelling ratio could be controlled by changing the alkyl chain length of HMG. In addition, the swelling ratio may be controlled by changing the modification ratio of the hydrophobic group. This swelling test indicated that HMG hydrogels still retained hydrophilic nature even after modification with hydrophobic segments.

\subsection{Adsorption test}

HMG hydrogel has many hydrophobic segments in its gel matrix. They can adsorb hydrophobic drugs. By observing the cross-sections of the hydrogels, it was confirmed that uranine was adsorbed not only to the hydrogel surface but also into the inside (date not shown). The amount of uranine adsorbed by HMG hydrogels with different alkyl chain lengths is shown in Figure 3. The adsorption amount of uranine increased as the alkyl chain length of the HMG molecules increased. This is because the increase in alkyl chain length intensifies the hydrophobic interaction acting between the hydrophobic drug and the HMG hydrogel. These results suggest that the HMG hydrogel can control the adsorption amount of hydrophobic drug by changing the alkyl chain length.

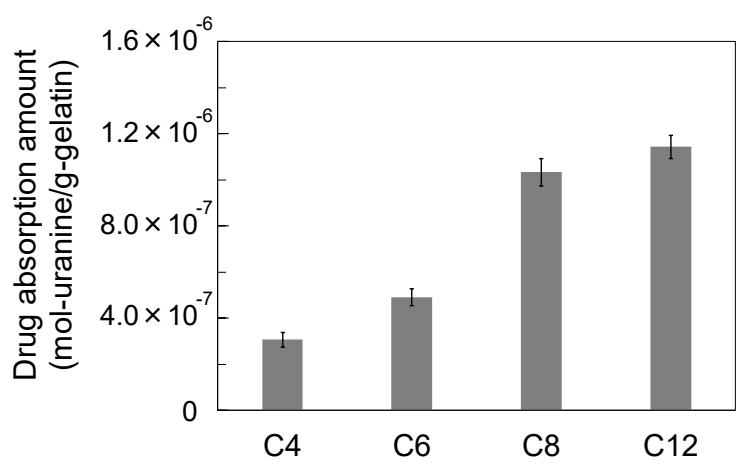

Figure 3. Amount of uranine adsorbed by HMG hydrogels with different alkyl chain length $(n=3)$.

\subsection{Desorption test}

In this experiment, the HMG hydrogel with the longest alkyl chain length $(\mathrm{C} 12)$ was used. About $35 \%$ of uranine was released just after soaking in the buffer to reach adsorption equilibrium (Figure 4). Uranine was further released when the hydrogel was transferred to a fresh buffer (6, 12 and 18h). In the body, kidneys filter waste materials involving drugs from body fluid and reduce their concentration. In such environment, the drugs would continue to be released from the hydrogel. Thus, these results suggest that HMG hydrogels can continue to release hydrophobic drugs in the body.

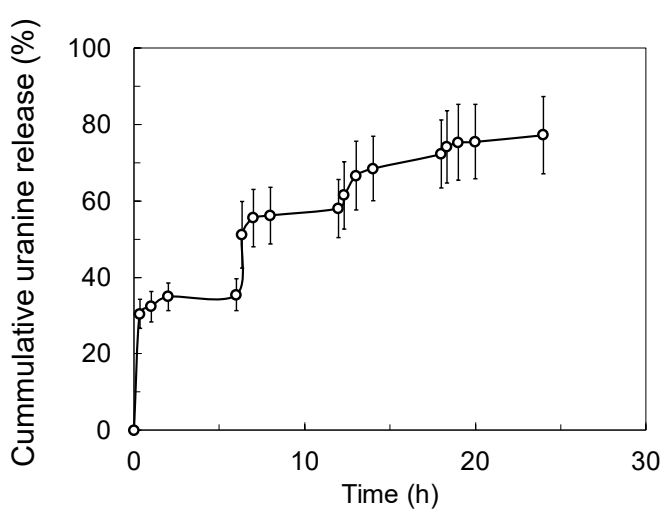

Figure 4. Uranine release profile from HMG hydrogel $(\mathrm{C} 12, n=3)$.

\section{Conclusion}

By modifying fatty aldehydes, gelatins with hydrophobic groups of different alkyl chain lengths (C4-C12) were synthesized, and physically cross-linked hydrogels were formed without using harmful chemical cross-linking agents. HMG hydrogels were able to control swelling characteristics and the adsorption amount of hydrophobic drugs by changing alkyl chain length. In addition, uranine was released from the HMG hydrogel $(\mathrm{C} 12)$ according to the adsorption equilibrium. Testing suggested that HMG hydrogels show potential as hydrophobic drug carriers. These advantages make HMG hydrogels a promising biomaterial for localized drug delivery systems.

\section{References}

Bhattarai, N., J. Gunn, and M. Zhang; "Chitosan-based Hydrogels for Controlled, Localized Drug Delivery," $A d v$. Drug Deliv. Rev., 62, 83-99 (2010)

Hoare, T. R. and D. S. Kohane; "Hydrogels in Drug Delivery: Progress and Challenges," Polymer, 49, 19932007 (2008)

Kidane, A. and P. P. Bhatt; "Recent Advances in Small Molecule Drug Delivery," Curr. Opin. Chem. Biol., 9, 347-351 (2005)

Larrañeta, E., S. Stewart, M. Ervine, R. Al-Kasasbeh, and R. F. Donnelly; "Hydrogels for Hydrophobic Drug Delivery. Classification, Synthesis and Applications," J. Funct. Biomater., 9, 13 (2018)

Li, Y., T. Su, S. Li, Y. Lai, B. He, and Z. Gu; "Polymeric Micelles with $\pi-\pi$ Conjugated Moiety on Glycerol Dendrimer as Lipophilic Segments for Anticancer Drug Delivery," Biomater. Sci., 2, 775-783 (2014)

Mauri, E., D. Naso, A. Rossetti, E. Borghi, E. Ottaviano, G. Griffini, M. Masi, and A. Sacchetti; "Design of Polymer-Based Antimicrobial Hydrogels through 
Physico-Chemical Transition," Mater. Sci. Eng. C, 103, 109791 (2019)

Morcol, T., A. Subramanian, and W. H. Velander; "Dotblot Analysis of the Degree of Covalent Modification of Proteins and Antibodies at Amino Groups," J. Immunol. Methods, 203, 45-53 (1997)

Nitta, K., A. Kimoto, and J. Watanabe; "Design and Synthesis of an Amphiphilic Graft Hydrogel Having Ahydrophobic Domain Formed by Multiple Interactions," Mater. Sci. Eng. C, 68, 65-69 (2016)

Nosrati, H., P. Barzegari, H. Danafar, and H. K. Manjili; "Biotin-Functionalized Copolymeric PEG-PCL Micelles for in vivo tumour-Targeted Delivery Of Artemisinin," Artif. Cells Nanomed. Biotechnol., 47, 104-114 (2019)

Saghazadeh, S., C. Rinold, M. Schot, S. S. Kashaf, F. Sharifi, E. Jalilian, K. Nuutila, G. Giatsidis, P. Mostafalu, H. Derakhshandeh, K. Yue, W. Swieszkowski, A. Memic, A. Tamayol, and A. Khademhosseini; "Drug Delivery Systems and Materials for Wound Healing Applications," Adv. Drug Deliv. Rev., 127, 138-166 (2018)

Senapati, S., A. K. Mahanta, S. Kumar, and P. Maiti; "Controlled Drug Delivery Vehicles for Cancer Treatment and Their Performance," Signal Transduct. Target Ther., 3, 1-19 (2018)

Yahia, Y., F. García-Villén, A. Djelad, L. S. Belaroui, R. Sanchez-Espejo, M. Sassi, A. López-Galindo, and C. Viseras; "Crosslinked Palygorskite-Chitosan Beads as Diclofenac Carriers," Appl. Clay. Sci., 180, 105169 (2019)

Yan, Q., L. Liu, T. Wang, and H. Wang; "A pHResponsive Hydrogel System Based On Cellulose and Dopamine with Controlled Hydrophobic Drug Delivery Ability and Long-Term Bacteriostatic Property," Colloid Polym. Sci., 297, 705-717 (2019)

Yoshizawa, K. and T. Taguchi; "Enhanced Bonding Strength of Hydrophobically Modified Gelatin Films on Wet Blood Vessels," Int. J. Mol. Sci., 15, 2142-2156 (2014)

Young, S., M. Wong, Y. Tabata, and A. G. Mikos; "Gelatin as a Delivery Vehicle for the Controlled Release of Bioactive Molecules," J. Control Release, 109, 256274 (2005)

Zhou, Y., G. Ma, S. Shi, D. Yang, and J. Nie; "Photopolymerized Water-soluble Chitosan-based Hydrogel as Potential Use in Tissue Engineering," Int. J. Biol. Macromol., 48, 408-413 (2011) 